ORIGINAL

\title{
The changes in treatment strategies in ABOi living donor liver transplantation for acute liver failure
}

\author{
Mitsuhiro Yasuda, Toru Ikegami, Daisuke Imai, Huanlin Wang, Yuki Bekki, Shinji Itoh, Tomoharu Yoshizumi, Yuji \\ Soejima, Ken Shirabe, and Yoshihiko Maehara \\ Department of Surgery and Science, Graduate School of Medical Sciences, Kyushu University, Fukuoka, Japan
}

\begin{abstract}
Introduction. Living donor liver transplantation (LDLT) using ABO-incompatible (ABOi) graft for acute liver failure (ALF) is a developing treatment modality. Methods. We reviewed the changes in our treatment strategies in applying ABOi LDLT for FH over our fourteen years of experience. Results. Five patients with ALF received LDLT in adults using ABOi grafts, with different but gradually renewed protocols. The etiologies for acute liver failure included autoimmune hepatitis $(n=3)$ and unknown $(n=2)$. The desensitization protocol for ABOi barrier included Case \# 1 ; local infusion (portal vein) + plasma exchange (PE), Case \#2 ; local infusion (hepatic artery) + rituximab + PE, Case \#3 and \#4 ; rituximab+PE, and Case \#5 ; rituximab + PE under high-flow continuous hemodiafiltration. Local infusion was abandoned since Case \#3, because Case \#1 had portal vein thrombosis resulting in graft necrosis and Case \#2 had hepatic artery dissection. The patients (Case \#2 and \#3), who received rituximab within 7 days before LDLT, experienced antibody-mediated rejection. Thus, the most recent protocol for ABOi-LDLT is that rituximab is given 2 weeks before LDLT, followed by high-flow continuous hemodiafiltration to obstacle hepatic encephalopathy until LDLT. The four patients except Case \#1 are doing well with good graft function over 3.8 \pm 3.7 years. Conclusion. Rituximab-based ABOi-LDLT, most-recently under high -flow hemodiafiltration for treating encephalopathy, is a feasible option for applying LDLT for ALF. J. Med. Invest. 62 : 184-187, August, 2015
\end{abstract}

Keywords : living donor liver transplantation, blood type incompatible, acute liver failure, rituximab, high-flow continuous hemodiafiltration.

\section{INTRODUCTION}

Although living donor LT (LDLT) has now become an option for treating patients with end-stage liver disease, its application may still be limited by the need for an appropriate living donor $(1,2)$. Under these circumstances, ABO incompatible (ABOi) LDLT has been practiced in Japan with recently improved outcomes by the recent invention and application of rituximab, a novel antiCD20 antibody terminating B-lymphocytes (3).

In applying rituximab in LDLT, however, it needs to be administered at least a few weeks before LDLT for its desirable effects. It is attributed that rituximab rituximab can only terminate CD-20 positive B-lymphocytes and it takes at least a few weeks to CD20 negative plasma cells to disappear spontaneously $(4,5)$. Thus, the application of ABOi-LDLT in emergent situation including acute liver failure (ALF) has not been a suitable option. In order to overcome this issue, we reported that high dose intravenous immunoglobulin (IVIG) was effective for treating antibody-mediated rejection (AMR) caused by remnant plasma cells after administration of rituximab (5). Progression of hepatic coma is the most significant determining prognostic factor in ALF, and therefore we recently started to use high-flow continuous hemodiafiltration (HF-CHDF) to treat hepatic coma and wait ABOi-LDLT for days to weeks after administration of rituximab (6).

In this article, we describe the historical changes in treatment strategies in applying ABOi-LDLT for ALF as a single institute experiences.

\section{Abbreviations}

ABO-incompatible (ABOi), acute liver failure (ALF), autoimmune hepatitis (AIH), antibody-mediated rejection (AMR), cyclosporine A (CsA), graft volume (GV), high-flow continuous hemodiafiltration (HF-CHDF), intravenous immunoglobulin (IVIG), living donor liver transplantation (LDLT), mycophenolate mofetil (MMF), model for end stage liver disease (MELD), plasma exchange (PE), standard liver volume (SLV), tacrolimus (Tac)

\section{MATERIALS AND METHODS}

\section{Patients}

Between May 1997 and December 2014, 486 LDLTs in adults including 30 LDLTs using ABOi grafts were performed at Kyushu University Hospital, Japan. Among them, 5 patients received ABOi LDLT for acute liver failure (Table 1). All the LDLTs were performed after obtaining full informed consent from all patients and approval by the Liver Transplantation Committee of Kyushu University. The basic surgical procedures and techniques were described previously $(1,7,8)$. All 5 patients received duct-to-duct biliary reconstruction. The mean follow-up period was $3.8 \pm 3.7$ years.

\section{Basic immunosuppression}

The basic immunosuppression induction regimen in ABOi LDLT involved the administration of tacrolimus (Tac) with mycophenolate mofetil (MMF) and steroids (Table 2). Currently, MMF is started 7 days before LDLT at a dose of $2 \mathrm{~g} /$ day, and increased to $3 \mathrm{~g} /$ day after LDLT, then decreased to $2 \mathrm{~g} /$ day once the blood calcineurin inhibitor level reaches an appropriate level. Tac is started within 3 days after LDLT once the kidney function has recovered. The target Tac level ranges between 12 to $15 \mathrm{ng} / \mathrm{ml}$ for the first post-LDLT month and is titrated down to 8 to $10 \mathrm{ng} / \mathrm{ml}$ for the next few months. When patients experience Tac associated complications, especially encephalopathy, Tac is converted to Cyclosporine A (CsA). The target CsA level ranges from 200 to 250

Received for publication January 9, 2015 ; accepted March 12, 2015.

Address correspondence and reprint requests to Toru Ikegami, MD, Department of Surgery and Science, Graduate School of Medical Sciences, Kyushu University, Fukuoka 812-8582, Japan and Fax : +81-92642-5482.

The Journal of Medical Investigation Vol. 622015 
Table 1. Patient demographics and survival data

\begin{tabular}{|c|c|c|c|c|c|c|c|c|c|c|c|}
\hline \multirow[b]{2}{*}{ No. } & \multicolumn{4}{|c|}{ Recipient } & \multicolumn{6}{|c|}{ Donor } & \multirow[b]{2}{*}{ Outcomes } \\
\hline & $\begin{array}{c}\text { Age/ } \\
\text { Gender }\end{array}$ & Etiology & $\begin{array}{c}\text { MELD } \\
\text { score }\end{array}$ & $\mathrm{ABO}$ & $\begin{array}{l}\text { Age/ } \\
\text { Gender }\end{array}$ & $\mathrm{ABO}$ & Relationship & $\begin{array}{l}\text { Graft } \\
\text { type }\end{array}$ & $\begin{array}{l}\text { GV } \\
(g)\end{array}$ & $\begin{array}{c}\text { GV/SLV } \\
(\%)\end{array}$ & \\
\hline$\# 1$ & $63 \mathrm{~F}$ & $\mathrm{AIH}$ & 15 & $\mathrm{O}$ & $32 \mathrm{M}$ & A & Son & Left & 650 & 66.1 & Dead $<1$ month \\
\hline$\# 2$ & $21 \mathrm{~F}$ & Unknown & 19 & B & $46 \mathrm{M}$ & $\mathrm{AB}$ & Mother & Right & 520 & 50.3 & Alive 9.1 years \\
\hline \#3 & $20 \mathrm{~F}$ & Unknown & 17 & $\mathrm{O}$ & $54 \mathrm{M}$ & A & Father & Right & 600 & 48.7 & Alive 7.8 years \\
\hline$\# 4$ & $68 \mathrm{~F}$ & $\mathrm{AIH}$ & 27 & A & $36 \mathrm{M}$ & $\mathrm{AB}$ & Son & Left & 499 & 41.6 & Alive 1.0 year \\
\hline \#5 & $51 \mathrm{~F}$ & AIH & 39 & $\mathrm{O}$ & $25 \mathrm{M}$ & $\mathrm{A}$ & Son & Left & 301 & 25.1 & Alive 10 months \\
\hline
\end{tabular}

autoimmune hepatitis (AIH), graft volume (GV), high-flow continuous hemodiafiltration (HF-CHDF), intravenous immunoglobulin (IVIG), living donor liver transplantation (LDLT), model for end stage liver disease (MELD), standard liver volume (SLV)

Table 2. Immunomodulation protocols in ABOi-LDLT

\begin{tabular}{cccccccc}
\hline No. & Local infusion & $\begin{array}{c}\text { Rituximab } \\
\text { (Pre-LDLT day) }\end{array}$ & $\begin{array}{c}\text { HF-CHDF } \\
\text { (days) }\end{array}$ & Splenectomy & $\frac{1 \text { Isoagglutinin titer }}{\text { before LDLT }}$ & $\begin{array}{c}\text { Plasma exchange } \\
\text { (Pre-, Post- LDLT) }\end{array}$ & $\begin{array}{c}\text { Basic } \\
\text { immunosuppression }\end{array}$ \\
\hline$\# 1$ & Yes (Portal) & - & - & Yes & $\underline{128->32}$ & x2,- & Tac, MMF, steroid \\
$\# 2$ & Yes (Artery) & Yes (-3) & - & Yes & $\underline{64->2}$ & x2, x5 & Tac, MMF, steroid \\
$\# 3$ & - & Yes (-3) & - & Yes & $\underline{2048->64}$ & x6, x5 & Tac, MMF, steroid \\
$\# 4$ & - & Yes (-14) & - & Yes & $\underline{32->32}$ & x19, - & CsA, MMF, steroid \\
$\# 5$ & - & Yes (-15) & Yes (11) & Yes & $\underline{512->128}$ & x13, - & Tac MMF, steroid \\
\hline
\end{tabular}

$\mathrm{ABO}$-incompatible (ABOi), cyclosporine A (CsA), high-flow continuous hemodiafiltration (HF-CHDF), living donor liver transplantation (LDLT), mycophenolate mofetil (MMF), tacrolimus (Tac)

$\mathrm{ng} / \mathrm{ml}$ for the first post-LDLT month and was titrated down to 100 to $150 \mathrm{ng} / \mathrm{ml}$ for the next few months. A gram of methylprednisolone is given after reperfusion, and tapered from $200 \mathrm{mg}$ to $40 \mathrm{mg}$ over 10 days, then switched to $20 \mathrm{mg}$ of oral Prednisolone and tapered off in 6 months after the LDLT.

Plasma exchange (PE) was performed to lower the isoagglutinin titer $\leq 64-128$. Splenectomy was performed during LDLT.

\section{Local infusion}

Local infusion via the portal vein or hepatic artery was used for between 2001 and 2006 (Table 2). A 16 G double lumen catheter was introduced from the umbilical vein or the mesenteric vein for portal vein orgastroduodenal artery for hepatic artery. Protease inhibitor (nafamostat mesilate, $200 \mathrm{mg} /$ day), Prostaglandin E1 (500 $\mathrm{mcg} /$ day) and methylprednisolone (50 mg/day) were given for 14 days after LDLT.

\section{Rituximab}

Rituximab ( $500 \mathrm{mg} /$ body) was given has been administered since the Case \#2 in 2005. As soon as the indication of the recipient with ALF was confirmed and the donor evaluation including volumetry and hematological/serological tests were completed, rituximab was administered. $\mathrm{PE}$ was held at least 8 hours after administration of rituximab and the number of $\mathrm{CD}-20$ positive lymphocytes were evaluated.

\section{High-flow continuous hemodiafiltration (HF-CHDF)}

HF-CHDF was performed as continuous venovenous hemodiafiltration to treat patients with marked comorbidities and general hemodynamic instability. Vascular access was created in the internal jugular vein or the femoral vein, using a 12-Fr flexible triplelumen catheter (Blood access catheter ${ }^{\mathrm{TM}}$, Arrow International Inc., Reading, PA, USA). A PMMA membrane hemofilter (Hemofeel $\mathrm{CH}^{\mathrm{TM}}$, Toray Medical Co. Ltd., Tokyo, Japan) was placed in the circuit. Nafamostat mesilate (Futhan ${ }^{\mathrm{TM}}$, Torii Pharmaceutical Co. Ltd., Tokyo, Japan) was used as anticoagulant, with the dose adjusted to maintain an activated coagulation time of 150-200 seconds. The operating conditions were set as follows : blood flow rate, 150-200 $\mathrm{ml} / \mathrm{min}$; dialysate flow rate, $8-20 \mathrm{~L} / \mathrm{hr}$; and filtration rate, 1.0-2.0 $\mathrm{L} / \mathrm{hr}$. The hemodiafiltration system was continuously monitored with a personal bedside console (JUN-500 ${ }^{\mathrm{TM}}$, Ube Medical Co. Ltd., Tokyo, Japan).

Values

The values are expressed as the mean \pm standard deviation.

\section{RESULTS}

\section{Recipient and donor data}

The five recipients who received ABOi-LDLT for ALF included all females, and the mean age was $44.6 \pm 19.3$ years. The etiologies for ALF included autoimmune hepatitis in three and unknown cause. The mean model for end-stage liver disease score was 23.4 \pm 7.7.

The donors included four males and one female, and the mean age, graft volume and graft volume/standard liver volume were, $38.6 \pm 9.1$ years, $514 \pm 91 \mathrm{~g}$, and $46.4 \pm 10.4 \%$, respectively (Table 1 ).

\section{Immunomodulation for $\mathrm{ABO}-\mathrm{LDLT}$}

The desensitization protocol for ABOi barrier included Case \#1 ; local infusion (portal vein) + plasma exchange (PE), Case \#2 ; local infusion (hepatic artery) + rituximab $+\mathrm{PE}$, Case \#3 and \#4 ; rituxi$\mathrm{mab}+\mathrm{PE}$, and Case \#5 ; rituximab $+\mathrm{PE}$ under high-flow continuous hemodiafiltration.

When Case \#1 was performed, rituximab was not available, and therefore local infusion of steroids, protease inhibitor and prostaglandin, under the control of isoagglutinin titer by $\mathrm{PE}$ was performed. Although isoagglutinin titer was controlled without applying PE after LDLT, the patient started to have diffuse thrombosis in the intrahepatic portal system 2 weeks after LDLT, resulting in graft necrosis and death. Although the definite reason for the diffuse portal thrombosis is unclear, we speculate that abrupt discontinuation of intra-portal protease inhibitor and prostaglandin might have caused increased coagulation activity in the portal system. 
Case \#2 received rituximab 3 days before LDLT and also had local infusion via the hepatic artery. She underwent re-laparotomy two times after LDLT for intraabdominal bleeding and hepatic artery dissection at the catheter tip. She also had AMR on day 7 and were treated by PEs. Since the initial two cases we abandoned local infusion treatment for its catheter associated complications.

Thus, the Case \#3 received rituximab without having local infusion despite her pre-transplant isoagglutinin titer was very high (x2048). Although she received PEs after LDLT, she had AMR on day 4. Her AMR was successfully treated by two session of highdose IVIG on day 5 and 10.

Since then, we changed our policy in ABOi-LDLT again, and planned to put rituximab 2 weeks before LDLT and apply HFCHDF to keep a patient away from brain death due to hepatic coma. Because the Case \#4 did not have rapid progressive encephalopathy, she did not received HF-CHDF and underwent scheduled LDLT 2 weeks after having rituximab. On the other hand, Case \#5 started to have grade II encephalopathy when she received rituximab, and therefore she was on HF-CHDF for 11 days before LDLT. The last two cases had fairly good post-transplant course.

\section{Graft outcomes}

The isoagglutinin titer after LDLT showed rebound elevation in the Case \#2 and \#3, both of which received rituximab within a week before LDLT, with deteriorating liver function tests indicating AMR. In both cases, CD-20 positive lymphocyte number was zero after LDLT despite their clinical AMR. Nevertheless, their AMR episodes were successfully treated by PE with or without IVIG.

Graft was lost only in the Case \#1, and therefore graft survival rate was $80 \%$ with good graft function over $3.8 \pm 3.7$ years.

\section{DISCUSSION}

Because there has been a very limited chance to perform liver transplantation from deceased donors in Japan, the use of ABOi donors are often required in LDLT (3-5). Thus, various methods have been implemented to allow these procedures, including local infusion (9). The theoretical basis of local administration of protease inhibitors, prostaglandin, and steroids is that the pathological findings of the failed ABOi liver graft show the features of hepatic disseminated intravascular coagulation (9). Although local infusion is a theoretically reasonable treatment of choice, there are problems associated with its application via the portal vein or hepatic artery includes catheter-related complications, and possible re-laparotomy for the removal of the catheters (10-12).

Rituximab, an anti-CD20 antibody, is a monoclonal antibody that specifically targets the CD20 surface antigen expressed onB lymphocytes, thus resulting in cell lyses. In the current series, the Case \#3 and \#4 were given Rituximab just 3 days before LDLT, and had rebound elevation of isoagglutinin titers with clinical AMR. Usui et al. (4) reported on its use as long as 3 weeks before the LDLT with successful outcomes. Egawa et al. (12) has reported that administration of rituximab earlier than 7 days before LDLT significantly depleted B-and memory B-lymphocytes. However in treating the patients with ALF, LDLT needs to be timely performed before they encounter advanced stages in hepatic encephalopathy and brain death due to their anhepatic state.

HF-CHDF uses much more volumes of buffer as much as $200 \mathrm{~L}$ per session and it efficiently removes more low and middle molecular weight toxic substances $(6,13,14)$. Yokoi, et al. (6) compared the clinical efficacy of HF-CHDF for treating patients with those of conventional treatments without HF-CHDF, and found that rate of recovery from coma was significantly higher in the HF-CHDF group $(70.2 \%)$ than in the non-HFCHDF group (44.2\%). The wider application of HF-CHDF has changed our strategies in applying ABOi-LDLT for ALF, because it inhibit the deterioration of hepatic encephalopathy and reserve days for plasma cells to be disappeared before emergent LDLT (15). Nevertheless, we think that IVIG is the last hope of treatment if encephalopathy were not controlled even by HF-CHDF and emergent LDLT could not be avoided (5).

IVIG might also be the significant factor in ABOi-LDLT. The proposed mechanisms of action of IVIG on the humoral reaction include B-cell apoptosis through the Fc-receptor dependent pathway, and the inhibition of alloreactive T-cell mediated or complementmediated allograft injury, although these possibilities have not been confirmed (16-18). The use of IVIG for induction therapy in ABOi liver transplantation has never been reported before, although a limited number of case reports have suggested the use of IVIG for severe AMR after ABOi LDLT (19). IVIG might have worked on for these B-lymphocytes, preventing AMR. In a deceased donor liver transplantation setting using ABOi donors, Urbani et al. (20) reported very favorable outcomes with the use of IVIG combined with plasma exchange and extracorporeal photopheresis.

The strategies for safer use of ABOi-LDLT grafts have been changes over years with refinements. Now our basic policy is rituximab given 2 weeks before LDLT for ALF, and HF-CHDF is applied to prevent deterioration of hepatic encephalopathy and brain death before LDLT. However if advance of encephalopathy could not be controlled by HF-CHDF, emergent LDLT should be performed and high-dose IVIG could be applied as a prophylaxis for AMR (Figure 1).

Living donor liver transplantation (+ Splenectomy)

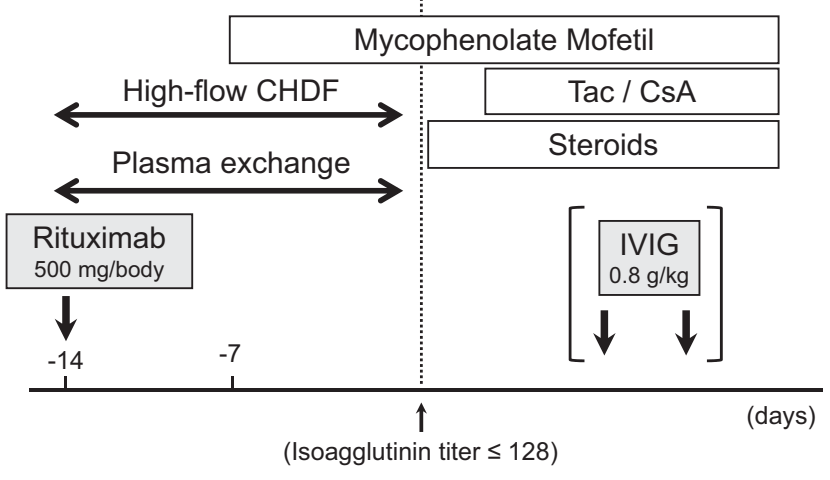

Figure 1. The current protocol for blood type incompatible living donor liver transplantation at Kyushu University Hospital.

In conclusion, refinements and revisions in ABOi-LDLT were performed over fourteen years of experiences, and currently rituximab-based ABOi-LDLT under HF-CHDF is our choice for treating deteriorating patients with ALF

\section{SOURCES OF SUPPORT}

This work was supported by a grant Grant-in-Aid for Scientific Research from the Ministry of Health, Labor and Welfare of Japan.

The authors have no conflicts of interest to disclose.

\section{AUTHOR CONTRIBUTIONS}

Mitsuhiro Yasuda : drafting of manuscript

Toru Ikegami : study concept and design 
Huanlin Wang : data collection

Yuki Bekki : data analysis

Daisuke Imai : data collection

Shinji Itoh : data collection

Norifumi Harimoto : data collection

Tomoharu Yoshizumi : data collection

Ken Shirabe : study design and critical revision of the manuscript

Yoshihiko Maehara : final approval of the manuscript

\section{REFERENCES}

1. Taketomi A, Kayashima H, Soejima Y, Yoshizumi T, Uchiyama H, Ikegami T, Yamashita Y, Harada N, Shimada M, Maehara $\mathrm{Y}$ : Donor risk in adult-to-adult living donor liver transplantation : impact of left lobe graft. Transplantation $87: 445-50$, 2009

2. Ikegami T, Shirabe K, Soejima Y, Yoshizumi T, Uchiyama H, Yamashita Y, Harimoto N, Toshima T, Yoshiya S, Ikeda T, Maehara Y : Strategies for successful left-lobe living donor liver transplantation in 250 consecutive adult cases in a single center. J Am Coll Surg 216 : 353-62, 2013

3. Egawa H, Teramukai S, Haga H, Tanabe M, Mori A, Ikegami T, Kawagishi N, Ohdan H, Kasahara M, Umeshita K : Impact of rituximab desensitization on blood-type-incompatible adult living donor liver transplantation : a Japanese multicenter study. Am J Transplant 14 : 102-14, 2014

4. Usui M, Isaji S, Mizuno S, Sakurai H, Uemoto S : Experiences and problems pre-operative anti-CD20 monoclonal antibody infusion therapy with splenectomy and plasma exchange for $\mathrm{ABO}$-incompatible living-donor liver transplantation. Clin Transplant $21: 24-31,2007$

5. Ikegami T, Taketomi A, Soejima Y, Yoshizumi T, Uchiyama H, Harada N, Iguchi T, Hashimoto N, Maehara Y : Rituximab, IVIG, and plasma exchange without graft local infusion treatment : a new protocol in ABO incompatible living donor liver transplantation. Transplantation $88: 303-7,2009$

6. Yokoi T, Oda S, Shiga H, Matsuda K, Sadahiro T, Nakamura $\mathrm{M}$, Hirasawa $\mathrm{H}$ : Efficacy of high-flow dialysate continuous hemodiafiltration in the treatment of fulminant hepatic failure. Transfus Apher Sci 40 : 61-70, 2009

7. Ikegami T, Shirabe K, Yamashita Y, Yoshizumi T, Harimoto N, Takeishi K, Tsujita E, Itoh S, Maehara Y : Small upper midline incision for living donor hemi-liver graft procurement in adults. J Am Coll Surg 219(3) : e39-43, 2014

8. Ikegami T, Soejima Y, Taketomi A, Yoshizumi T, Harada N, Uchiyama H, Shimada M, Maehara : Explanted portal vein grafts for middle hepatic vein tributaries in living-donor liver transplantation. Transplantation $84: 836-41,2007$

9. Tanabe M, Shimazu M, Wakabayashi G, Hoshino K, Kawachi S, Kadomura T, Seki H, Morikawa Y, Kitajima M : Intraportal infusion therapy as a novel approach to adult $\mathrm{ABO}$-incompatible liver transplantation. Transplantation $73: 1959-61,2002$

10. Egawa H, Ohdan H, Haga H, Tsuruyama T, Oike F, Uemoto $\mathrm{S}$, Ozawa $\mathrm{K}$ : Current status of liver transplantation across ABO blood-type barrier. J Hepatobiliary Pancreat Surg 15 : 131-8, 2008

11. Egawa H, Teramukai S, Haga H, Tanabe M, Fukushima M, Shimazu M : Present status of ABO-incompatible living donor liver transplantation in Japan. Hepatology $47: 143-52,2008$

12. Egawa $\mathrm{H}$, Ohmori K, Haga H, Tsuji H, Yurugi K, MiyagawaHayashino A, Oike F, Fukuda A, Yoshizawa J, Takada Y, Tanaka K, Maekawa T, Ozawa K, Uemoto S : B-cell surface marker analysis for improvement of rituximab prophylaxis in $\mathrm{ABO}$-incompatible adult living donor liver transplantation. Liver Transpl $13: 579-88,2007$

13. Kubota T, Sekido H, Takeda K, Morioka D, Tanaka K, Endo I, Togo S, Saitoh S, Numata K, Tanaka K, Sekihara H, Matsunami H, Tanaka K, Shimada H : Acute hepatic failure with deep hepatic coma treated successfully by high-flow continuous hemodiafiltration and living-donor liver transplantation : a case report. Transplant Proc 35 : 394-6, 2003

14. Inoue $K$, Watanabe $T$, Hirasawa $H$, Yoshiba $M$ : Liver support systems as perioperative care in liver transplantation-historical perspective and recent progress in Japan. Minerva Gastroenterol Dietol 56 : 345-53, 2010

15. Ikegami T, Taketomi A, Soejima Y, Yoshizumi T, Sanefuji K, Kayashima H, Shimada M, Maehara Y : Living donor liver transplantation for acute liver failure : a 10-year experience in a single center. J Am Coll Surg 206 : 412-8, 2008

16. Jordan SC, Vo AA, Peng A, Toyoda M, Tyan D : Intravenous gammaglobulin (IVIG) : a novel approach to improve transplant rates and outcomes in highly HLA-sensitized patients. Am J Transplant 6 : 459-66, 2006

17. Sonnenday CJ, Warren DS, Cooper M, Samaniego M, Haas M, King KE, Shirey RS, Simpkins CE, Montgomery RA : Plasmapheresis, CMV hyperimmune globulin, and anti-CD20 allow $\mathrm{ABO}$-incompatible renal transplantation without splenectomy. Am J Transplant 4 : 1315-22, 2004

18. Jordan S, Cunningham-Rundles $\mathrm{C}, \mathrm{McEwan} \mathrm{R}$ : Utility of intravenous immune globulin in kidney transplantation : Efficacy, safety, and cost implications. Am J Transplat $3: 653-64,2003$

19. Ikegami T, Taketomi A, Soejima Y, Iguchi T, Sanefuji K, Kayashima H, Yoshizumi T, Harada N, Maehara Y : Successful $\mathrm{ABO}$ incompatible living donor liver transplantation in a patient with high isoagglutinin titer using high-dose intravenous immunoglobulin. Transplant Proc 39 : 3491-4, 2007

20. Urbani L, Mazzoni A, Bianco I, Grazzini T, De Simone P, Catalano G, Montin U, Petruccelli S, Morelli L, Campani D, Pollina L, Biancofiore G, Bindi L, Tascini C, Menichetti F, Scatena $\mathrm{F}$, Filipponi $\mathrm{F}$ : The role of immunomodulation in $\mathrm{ABO}$-incompatible adult liver transplant recipients. J Clin Apher 23 : 55-62, 2008 